\title{
Variation on a Theme: Alternative to Plastic Bag in ALPPS Procedures. Feasibility and Clinical Safety of COVA+ ${ }^{\mathrm{TM}}$ Membrane in ALPPS Procedures
}

\author{
Raffaele Brustia $^{1,3}$ - Olivier Scatton ${ }^{1,3}$ - Olivier Soubrane $e^{2,4}$
}

Published online: 28 December 2015

(C) Société Internationale de Chirurgie 2015

Sir,

We read with interest the alternative technique proposed by Ettorre et al. [1] reported after its use on three patients undergoing ALPPS procedure, to prevent adhesions. The idea relies on the use of the gallbladder peritoneum, stitched on the cut surface of the deportalized liver. Furthermore, the authors propose the use of the falciform ligament as a second patch to cover the cut surface of the left liver. The technique is simple and cost-free. However, the gallbladder absence (e.g., previous cholecystectomy) and the case of tumoral process starting from, or involving, the gallbladder can be troublesome. Since the first ALPPS report [2], many alternatives on technical details has been described: among them, the depth of liver transection until the vena cava (VC) or not [3]. Our policy is to follow the original description and continue the transection beyond the hilar plate until the $\mathrm{VC}$, where most of adhesions appear. Unfortunately, neither the gallbladder peritoneum

Olivier Soubrane

olivier.soubrane@aphp.fr

Raffaele Brustia

raffaele.brustia@gmail.com

1 Department of Hepatobiliary Surgery and Liver Transplantation, Hôpital Pitié-Salpêtrière, Assistance Publique-Hôpitaux de Paris, 47-83 Boulevard de l'Hôpital, 75013 Paris, France

2 Department of Hepatobiliary Surgery and Liver Transplantation, Hôpital Beaujon, Assistance PubliqueHôpitaux de Paris, 100 Boulevard du Général Leclerc, 92110 Clichy, France

3 Université Pierre et Marie Curie, Paris, France

4 Université Diderot, Paris, France nor the falciform ligament usually totally covers the liver cut surface, while the acellular collagen membrane that we used has larger and standard dimensions, adapted to cover the entire transected plane. Anyhow, the proposal from Ettorre et al. [1] can be ascribed - as well as ours [4, 5]— to the broader category of "variations on a theme." The debate around the ALPPS procedure is not finished yet, and the last word is far to be written.

Compliance with ethical standards

Conflict of interest The authors declare no conflict of interest.

\section{References}

1. Ettorre GM, Guglielmo N, Felli E, Meniconi RL, Colasanti M, Lepiane P, Santoro R, Vennarecci G (2015) Is there still a room to improve the safety of ALPPS procedure? A new technical note. Eur J Surg Oncol 41:1556-1557

2. Schnitzbauer AA, Lang SA, Goessmann H et al (2012) Right portal vein ligation combined with in situ splitting induces rapid left lateral liver lobe hypertrophy enabling 2-staged extended right hepatic resection in small-for-size settings. Ann Surg 255:405-414

3. Petrowsky H, Györi G, de Oliveira M, Lesurtel M, Clavien PA (2015) Is partial-ALPPS safer than ALPPS? A single-center experience. Ann Surg 261:e90-e92

4. Brustia R, Scatton O, Soubrane O (2015) Variation on a theme: alternative to plastic bag in ALPPS procedures: feasibility and clinical safety of COVA $+{ }^{\mathrm{TM}}$ membrane in ALPPS procedures. World J Surg 39:3023-3027. doi:10.1007/s00268-015-3209-Z

5. Brustia R, Scatton O, Perdigao F, El-Mouhadi S, Cauchy F, Soubrane O (2013) Vessel identifications tags for open or laparoscopic associating liver partition and portal vein ligation for staged hepatectomy. J Am Coll Surg 217:e51-e55 